\title{
High geocentric velocity meteor ablation
}

\author{
K. A. Hill, L. A. Rogers`, and R. L. Hawkes \\ Physics Department, Mount Allison University, 67 York St., Sackville, NB E4L 1E6, Canada \\ e-mail: kahll@mta.ca; lroge014@uottawa.ca; rhawkes@mta.ca
}

Received 13 March 2005 / Accepted 20 July 2005

\begin{abstract}
Interstellar origin meteoroids have now been detected using radar, image intensified video, large aperture radar and space dust impact techniques. Dynamical and radiation production mechanisms will eject some meteoroids from other planetary systems into orbits which will impact Earth with high geocentric velocities. In this paper we numerically model the ablation of high geocentric velocity $\left(71\right.$ to $\left.500 \mathrm{~km} \mathrm{~s}^{-1}\right) \mathrm{meteors} \mathrm{in}$ order to predict the heights, light curves and trail lengths to be expected. We modeled three compositions and structures: asteroidal, cometary and porous cometary. Meteoroid masses ranging from $10^{-6}$ to $10^{-13} \mathrm{~kg}$ were used in the model. As expected, these high geocentric velocity meteors, when compared to other meteors, ablate higher in the atmosphere. For example a $300 \mathrm{~km} \mathrm{~s}^{-1}$ cometary structure meteor of mass $10^{-9} \mathrm{~kg}$ will have a peak luminosity at about $190 \mathrm{~km}$. They will also have significantly longer trail lengths. The same $300 \mathrm{~km} \mathrm{~s}{ }^{-1}, 10^{-9} \mathrm{~kg}$ cometary meteor would be within 2 mag of its peak brightness for a vertical displacement of $60 \mathrm{~km}$ if incident at a zenith angle of $45^{\circ}$. The peak light intensity of these high geocentric velocity meteors changes only slowly with velocity. Although the incident kinetic energy per unit time increases dramatically, this is largely offset by a decrease in the optical luminous efficiency in this velocity regime according to our luminous efficiency model. The $300 \mathrm{~km} \mathrm{~s}^{-1}, 10^{-9} \mathrm{~kg}$ cometary meteor would have an absolute meteor magnitude at peak luminosity of about +8.5 mag. Our results suggest that at least those high geocentric velocity meteors larger than about $10^{-8} \mathrm{~kg}$ should be observable with current meteor electro-optical technology although there may be observational biases against their detection. The results of this paper can be used to help optimize a search strategy for these very high geocentric velocity meteors.
\end{abstract}

Key words. meteors, meteorids - methods: numerical

\section{Introduction}

It has now become well established that meteors of interstellar origin have been detected by image intensified charge coupled device (CCD), radar, large aperture radar and space detector techniques (Hawkes et al. 1999). While most such meteors will have pre-Solar System velocities which are of the order of the random velocities of stars in the solar neighbourhood (typically 10 to $40 \mathrm{~km} \mathrm{~s}^{-1}$ ), occasionally we would expect that interstellar origin meteoroids will have much higher encounter velocities. There is some evidence for the radar detection of meteors with high geocentric velocities. Taylor et al. (1996) found that nearly $1 \%$ of the meteors observed with the Advanced Meteor Orbit Radar (AMOR) had apparent velocities of greater than $100 \mathrm{~km} \mathrm{~s}^{-1}$. This leaves open the possibility of a significant number of meteors with high geocentric velocities. No optical detections with very high geocentric velocities exist, and as pointed out by Hawkes et al. (1999) even most heliocentric hyperbolic orbits result in geocentric velocities less than $72 \mathrm{~km} \mathrm{~s}^{-1}$ due to the statistics of likely encounter conditions. However the sample of interstellar origin meteors detected

^ Current address: Physics Department, University of Ottawa, ON Canada. optically is small, and the observational velocity characteristics are not well established.

While a number of interstellar meteor production mechanisms are possible, perhaps the most likely methods for injection of meteoroids into interstellar space are radiation pressure forces from massive stars and dynamical interactions involving planets around stars with significant dust clouds. Brophy (1991) provided an early analysis of the flux of interstellar material expected and detected at the Earth. Hawkes \& Woodworth (1997) used a very simple model to show that radiation pressure from massive young stars might produce the interstellar meteoroids detected using space, radar and optical techniques. A recent study of meteoroid ejection velocities from pre-main sequence stellar systems taking into account radiation pressure, Poynting-Robertson and gravitational forces suggests that in some cases small meteoroids may be produced with velocities of the order of at least several hundred $\mathrm{km} \mathrm{s}^{-1}$ (Quirt \& Hawkes 2005). Recently Murray et al. (2004) have considered production mechanisms from AGB stars, young stellar objects such as $\mathrm{T}$ Tauri stars, and debris disks around Vega-like stars. They have also considered the survival in interstellar space of such material. Baggaley (2000) suggests that a significant fraction of the AMOR observed interstellar meteoroids may come from a single source $\beta$-Pictoris. A possible 
Table 1. Thermal ablation parameters for Eqs. (1)-(7).

\begin{tabular}{ccc}
\hline \hline Symbol & Definition & Numerical value \\
\hline$h$ & height & - \\
$v$ & velocity & - \\
$m$ & mass & - \\
$T$ & meteoroid temperature & - \\
$z$ & zenith angle & $45^{\circ}$ \\
$\rho_{\mathrm{a}}$ & atmospheric mass density & - \\
$\rho_{\mathrm{m}}$ & meteoroid mass density & - \\
$\Gamma$ & drag coefficient & 1.0 \\
$A$ & shape factor & 1.21 \\
$\Lambda$ & heat transfer coefficient & 1.0 \\
$\sigma$ & Stefan-Boltzmann constant & $5.67 \times 10^{-8} \mathrm{~W} \mathrm{~m} \mathrm{~m}^{-2} \mathrm{~K}^{-4}$ \\
$\epsilon$ & emissivity & 0.9 \\
$T_{\mathrm{a}}$ & effective atmospheric temperature & $280^{\circ} \mathrm{K}$ \\
$c$ & specific heat of meteoroid & $1200 \mathrm{~J} \mathrm{~K}^{-1} \mathrm{~kg}^{-1}$ \\
$L$ & latent heat of fusion plus vaporization & $6.0 \times 10^{6} \mathrm{~J} \mathrm{~kg}^{-1}$ \\
$P_{\mathrm{V}}$ & saturated vapor pressure of meteoroid & - \\
$\mu$ & mean molecular mass of ablated material & - \\
$k$ & Boltzmann constant & $1.381 \times 10^{-23} \mathrm{~J} \mathrm{~K}^{-1}$ \\
$C_{\mathrm{A}}$ & Clausius Clapeyron parameter & 10.6 \\
$C_{\mathrm{B}}$ & Clausius Clapeyron parameter & $13500 \mathrm{~K}^{-1}$ \\
$\tau_{I}$ & luminous efficiency factor & - \\
\hline
\end{tabular}

production mechanism has been suggested by Krivova \& Solanki (2003). Baggaley \& Neslusan (2002) have examined how a stream of particles from a single interstellar source would encounter the Earth in terms of observed velocity distributions. Murray et al. (2004), however, have recently suggested that it is unlikely that radar detectable interstellar meteors are produced by $\beta$-Pictoris. Issues of the subsequent destruction of these interstellar meteoroids (see e.g. Jones et al. 1997; Murray et al. 2004), or their velocity alteration in interstellar space may mean that very few would be expected to survive to Earth. The possibility exists, however, of at least a small sample of very high geocentric velocity material.

In this paper we model the heights, trail lengths and luminosities expected from high geocentric velocity meteor ablation in the Earth's atmosphere. We have modeled meteoroids with three different compositions and structures; asteroidal type meteoroids, cometary structure meteoroids, and porous cometary material. This does not presume that significant numbers of meteoroids with such velocities have been shown to exist. Rather, by providing predictions of their observational characteristics we hope to provide direction to those who seek to prove or disprove the existence of detectable numbers of high geocentric velocity interstellar origin meteoroids. In a short preliminary paper (Rogers et al. 2005a) we first considered the ablation of high geocentric velocity meteors. The current paper extends the velocity regime, expands the number of composition-structure models, and provides more complete information on the height, trail length, and light curve results. Also, we provide here for the first time the details of the numerical models used.

\section{Theory}

Due to the fact that thermal ablation theory is well established in meteor physics (Öpik 1958; McKinley 1961; Hawkes \& Jones 1975; Nicol et al. 1985; Ceplecha et al. 1998; Fisher et al. 2000), we will only review the ablation equations. The parameters within the equations are defined in Table 1 and their values (where applicable) are given.

The rate of change of height of a meteoroid entering the Earth's atmosphere follows from simple geometry;

$\frac{\mathrm{d} h}{\mathrm{~d} t}=-v \cos z$

As the meteoroid travels through the atmosphere, collisions with atmospheric constituents will cause its momentum to decrease. If we equate the momentum per second lost by the meteoroid to the momentum per second gained by the atmospheric particles, we can obtain the meteoroid deceleration equation;

$\frac{\mathrm{d} v}{\mathrm{~d} t}=-\frac{\Gamma A}{m^{1 / 3} \rho_{\mathrm{m}}^{2 / 3}} \rho_{\mathrm{a}} v^{2}$

To obtain the rate of change of temperature of the meteoroid, we assume that we have a homogeneous meteoroid experiencing an isotropic heat flux. We also make the assumption that the meteoroid undergoes isothermal heating. The heat balance equation per cross-sectional area is then given by

$\frac{1}{2} \Lambda \rho_{\mathrm{a}} v^{3}=4 \sigma \epsilon\left(T^{4}-T_{\mathrm{a}}^{4}\right)+\frac{c m^{1 / 3} \rho_{\mathrm{m}}^{2 / 3}}{A} \frac{\mathrm{d} T}{\mathrm{~d} t}-\frac{L}{A}\left(\frac{\rho_{\mathrm{m}}}{m}\right)^{2 / 3} \frac{\mathrm{d} m}{\mathrm{~d} t} \cdot(3)$

The term on the left hand side of Eq. (3) represents the meteoroid heat flux received per unit cross-sectional area from the impinging molecules. The terms on the right hand side of Eq. (3) represent heat lost due to thermal radiation, meteoroid heating, and vaporization.

If we solve Eq. (3) for $\mathrm{d} T / \mathrm{d} t$, we obtain

$\frac{\mathrm{d} T}{\mathrm{~d} t}=\frac{A}{c m^{1 / 3} \rho_{\mathrm{m}}^{2 / 3}}\left(\frac{1}{2} \Lambda \rho_{\mathrm{a}} v^{3}-4 \sigma \epsilon\left(T^{4}-T_{\mathrm{a}}^{4}\right)+\frac{L}{A}\left(\frac{\rho_{\mathrm{m}}}{m}\right)^{2 / 3} \frac{\mathrm{d} m}{\mathrm{~d} t}\right)$.

Previous studies of meteoroid ablation have assumed that meteoroids lose mass only when they have reached their boiling 
point (e.g. McKinley 1961; Hawkes \& Jones 1975; Nicol et al. 1985; Fisher et al. 2000). That assumption, however, neglected the small amount of evaporation that occurs as the meteoroid is heating before reaching its boiling point. To account for this small amount of mass loss, we have instead used the vapor pressure to model the ablation of the meteoroid following the method of Bronshten (1983) and Adolfsson et al. (1996).

$\frac{\mathrm{d} m}{\mathrm{~d} t}=-4 A\left(\frac{m}{\rho_{\mathrm{m}}}\right)^{2 / 3} P_{\mathrm{V}}(T)\left(\frac{\mu}{2 \pi k T}\right)^{1 / 2}$.

The saturated vapor pressure is determined using the Clausius-Clapeyron equation;

$\log _{10} P_{\mathrm{V}}(T)=C_{\mathrm{A}}-\frac{C_{\mathrm{B}}}{T}$.

We have adopted the thermal parameters $\left(C_{\mathrm{A}}\right.$ and $\left.C_{\mathrm{B}}\right)$ used by Adolfsson et al. (1996); see Table 1 for the values.

The light intensity of the meteoroid was calculated using the normal luminous equation

$I=-\frac{\tau_{I}}{2} \frac{\mathrm{d} m}{\mathrm{~d} t} v^{2}$

The luminous efficiency factor, $\tau_{I}$, represents the fraction of the meteoroid's kinetic energy which has been converted into light energy in the visual range.

The value of the shape factor $A, 1.21$, represents a spherical meteoroid. We used the values employed by Fisher et al. (2000) for $\Gamma, \Lambda, \epsilon, T_{\mathrm{a}}, c$ and $L$.

When modeling the ablation of a meteoroid, the atmospheric mass density is a very significant quantity. Older models used simplified constant scale height atmospheres, but we have used a more recent model; the NASA Mass-Spectrometer-Incoherent-Scatter Extended (MSISE) 1990 atmospheric model (Hedin 1987, 1991). Since there has been evidence that the atmospheric mass density varies both with the season and the solar cycle (Ellyett 1977; Śimek \& Pecina 2002), we have done an extensive averaging process to ensure that our values represent a reasonable mean behaviour. We have been guided by the equations presented in Rogers et al. (2005b) but since the mass density at higher altitudes is very important to this work, we have slightly refined their atmospheric profile. The dependence of the averaged atmospheric mass density with the height above the surface of the earth is displayed in Fig. 1.

Equations (1), (2), (4), and (5) form a system of coupled differential equations. They were solved numerically using a 4 th order Runge-Kutta numerical method. We used a semiadaptive step size as described in Rogers et al. (2005b). The initial (and maximum) step size was chosen so that the meteoroid traveled $5 \mathrm{~cm}$ between each iteration. As the meteoroid began to experience significant mass loss, the step size was reduced; this variable step size was taken to be proportional to the meteoroid mass while inversely proportional to the meteoroid velocity and rate of change of mass. During the final stages of meteoroid ablation, the step size was held constant at its value at the meteoroid's peak light intensity. The model

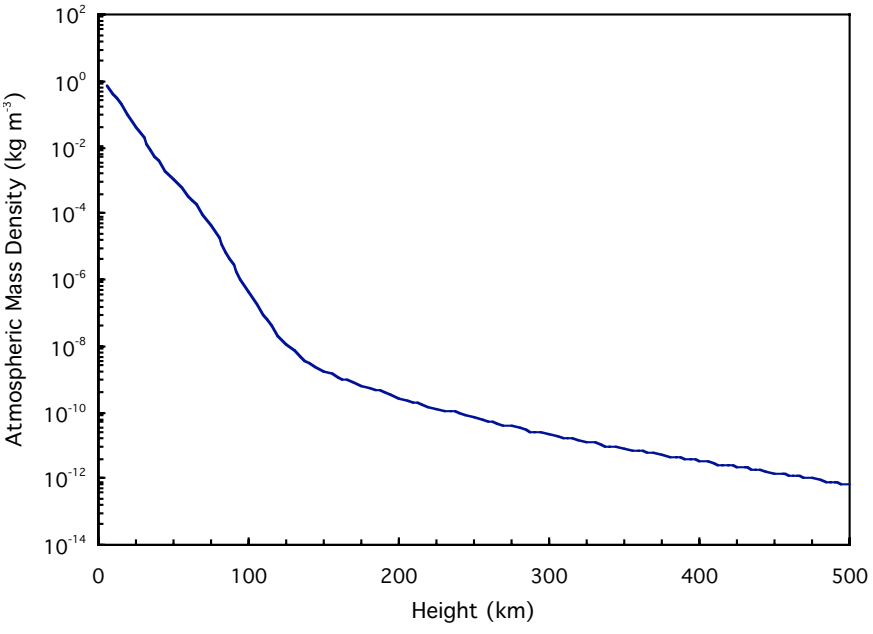

Fig. 1. Atmospheric mass density as a function of height, based on averaged data from the MSISE-90 atmospheric model.

began with the meteoroid at an altitude of $500 \mathrm{~km}$ and a temperature of $250 \mathrm{~K}$ and it terminated when the meteoroid mass had decreased to 0.00001 of its initial value or when the meteoroid velocity had decreased to below $6.2 \mathrm{~km} \mathrm{~s}^{-1}$ (at which point the light intensity decreased to zero).

\section{Meteor luminosity}

Luminosity occurs in meteors as a result of decay of excited atomic (and a few molecular) states following collisions between ablated meteor atoms and atmospheric constituents (Ceplecha et al. 1998). We can express this in the form of the normal luminous equation (Eq. (7)).

One can easily see that the value of $\tau_{I}$ is of great importance in the results of Eq. (7).

Until quite recently, $\tau_{I}$ had usually been assumed to increase linearly with velocity. This was previously suggested by Whipple (1938). A direct observational study done by Verniani (1965) seemed to produce results indicating that $\tau_{I}$ had the form

$\tau_{I}=\tau_{0} v$

where, if all quantities were expressed SI units, $\tau_{0}$ has the value of $5.25 \times 10^{-8}$. One cannot rely on this equation, however, since Verniani did not account for the total effect of meteoroid fragmentation when determining $\tau_{0}$ (Jones \& Halliday 2001).

More recently, Jones \& Halliday (2001) have done work dealing with the luminous efficiency for higher velocity meteors $\left(20 \mathrm{~km} \mathrm{~s}^{-1} \leq v \leq 100 \mathrm{~km} \mathrm{~s}^{-1}\right)$. They define a universal excitation coefficient $\zeta$ by writing

$\frac{1}{2} \tau_{I} \mu v^{2}=\epsilon \zeta$

where $\epsilon$ is a mean excitation energy and $\mu$ is an atomic mass. It turns out that the excitation coefficient $\zeta$ is a sum of excitation probabilities over collisions. If one rearranges Eq. (9) for $\tau_{I}$, one obtains

$\tau_{I}=2\left(\frac{\epsilon}{\mu}\right) \frac{\zeta}{v^{2}}$ 
There are two important characteristics to note in the above equation: i) The luminous efficiency factor $\tau_{I}$ depends on the ratio of the mean excitation energy to the atomic mass $(\epsilon / \mu)$ for important meteoric species, and ii) One must know the value of the excitation coefficient $\zeta$ to compute $\tau_{I}$. Although we do see some evidence of atmospheric radiation, the majority of the light comes from meteoric atoms. According to Bronshten (1983), less than $3 \%$ of the visible light from meteors is due to excitation of atmospheric molecules.

We used meteor spectra data given by Ceplecha et al. (1998) to compute $\epsilon / \mu$ for the meteoric atoms and ions which produce the greatest light intensity in the visual regime. We then obtained the mean value of $\epsilon / \mu, 7.668 \times 10^{6} \mathrm{~J} \mathrm{~kg}^{-1}$, to represent $\epsilon / \mu$ for a typical meteor used in our model.

The excitation coefficient $\zeta$ was split into several regimes:

(i) $v \leq 20 \mathrm{~km} \mathrm{~s}^{-1}$

In this regime, we used luminous efficiency data given by Becker \& Friichtenicht (1971) for iron and $\epsilon / \mu$ for iron to compute the universal excitation coefficient $\zeta$.

$$
\begin{aligned}
\zeta= & \left(-2.1887 \times 10^{-9}\right) v^{2}+\left(4.2903 \times 10^{-13}\right) v^{3} \\
& +\left(-1.2447 \times 10^{-17}\right) v^{4} .
\end{aligned}
$$

When $v=6.2 \mathrm{~km} \mathrm{~s}^{-1}$, Eq. (11) yields $\zeta=0$; in other words, the excitation probability over all collisions is zero, so no light will be produced.

(ii) $20 \mathrm{~km} \mathrm{~s}^{-1} \leq v \leq 60 \mathrm{~km} \mathrm{~s}^{-1}$

Jones \& Halliday (2001) used a luminous efficiency approximation for iron given by Friichtenicht \& Becker (1973) and the relative intensities for the Fe multiplets given by Boitnott \& Savage (1972) to obtain the following equation for the excitation coefficient:

$\zeta=0.01333 v^{1.25}$.

Equation (12) was initially only considered valid for velocities between $20 \mathrm{~km} \mathrm{~s}^{-1}$ and $46 \mathrm{~km} \mathrm{~s}^{-1}$, but when it was extrapolated to $60 \mathrm{~km} \mathrm{~s}^{-1}$, it was in quite satisfactory agreement with experimental data.

(iii) $60 \mathrm{~km} \mathrm{~s}^{-1} \leq v \leq 100 \mathrm{~km} \mathrm{~s}^{-1}$

The encouraging results from extrapolating the excitation coefficient to $60 \mathrm{~km} \mathrm{~s}^{-1}$ in the previous regime encouraged Jones \& Halliday to extend it to velocities above $60 \mathrm{~km} \mathrm{~s}^{-1}$. From $60 \mathrm{~km} \mathrm{~s}^{-1} \leq v \leq 100 \mathrm{~km} \mathrm{~s}^{-1}$, values for $\zeta$ were determined based on the Boitnott-Savage crosssections for this region. These values of $\zeta$ were used in our model according to the following regression.

$$
\begin{aligned}
\zeta= & (-12.835)+\left(6.7672 \times 10^{-4}\right) v \\
& +\left(-1.163076 \times 10^{-8}\right) v^{2} \\
& +\left(9.191681 \times 10^{-14}\right) v^{3} \\
& +\left(-2.7465805 \times 10^{-19}\right) v^{4}
\end{aligned}
$$

(iv) $v \geq 100 \mathrm{~km} \mathrm{~s}^{-1}$

Above a velocity of $100 \mathrm{~km} \mathrm{~s}^{-1}$, we extended Jones \& Halliday's excitation coefficient data linearly using data

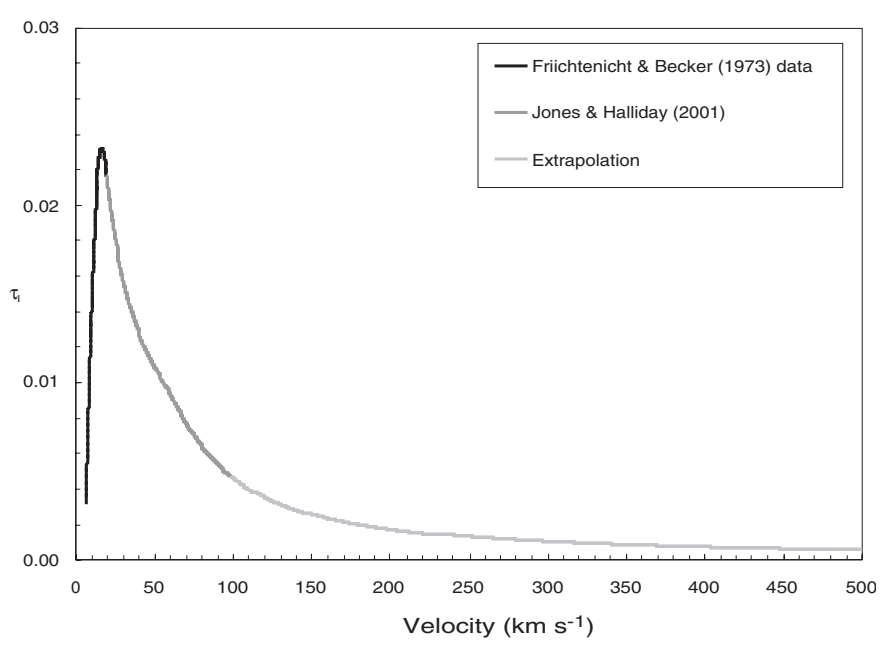

Fig. 2. Velocity dependence of the luminous efficiency factor, $\tau_{I}$, for radiation in the visible region of the electromagnetic spectrum.

points from between $v=95 \mathrm{~km} \mathrm{~s}^{-1}$ and $v=100 \mathrm{~km} \mathrm{~s}^{-1}$ as the basis for the linear extrapolation (given below in Eq. (14)). This will be the region which is least certain since there is no observational data available to compare with this extrapolation.

$\zeta=(1.615)+\left(1.3725 \times 10^{-5}\right) v$.

Although the excitation coefficient in all velocity regimes has been derived using luminous efficiency data and theoretical cross-sections for iron, through Eq. (10) we have used $\epsilon / \mu$ for a typical meteoroid to determine the luminous efficiency of a meteoroid consisting not solely of iron.

Figure 2 shows a graph of luminous efficiency factor vs. velocity throughout the aforementioned velocity regimes using the relationships for the excitation coefficient given above and the theory developed by Jones \& Halliday (2001).

\section{Results}

The purpose of this study was to examine the ablation of very high velocity meteoroids. Geocentric velocities of $71,80,90$, $100,125,150,175,200,300,400$, and $500 \mathrm{~km} \mathrm{~s}^{-1}$ were considered. The velocity of $71 \mathrm{~km} \mathrm{~s}^{-1}$ lies just below the hyperbolic limit, while the other velocities examined are representative of either interstellar meteoroids or meteoroids which have been accelerated by mechanisms within the solar system. Meteoroid masses ranging from $10^{-6}$ to $10^{-13} \mathrm{~kg}$ (in increments of a factor of 10) were employed for each of the three structures (asteroidal, cometary and porous). The asteroidal meteoroids were assumed to have a molecular mass of 50 amu and a density of $3300 \mathrm{~kg} \mathrm{~m}^{-3}$, while the cometary meteoroids were assumed to have a molecular mass of 20 amu and a density of $1000 \mathrm{~kg} \mathrm{~m}^{-3}$, and the porous cometary meteoroids a molecular mass of $20 \mathrm{amu}$ and a density of $300 \mathrm{~kg} \mathrm{~m}^{-3}$.

The meteor heights at peak light intensity $\left(H_{\max }\right)$ are presented in Fig. 3 (asteroidal structure), Fig. 4 (cometary structure) and Fig. 5 (porous structure). These heights are observed to increase with velocity, the increase being more dramatic for smaller initial meteoroid masses. The observed dependence 


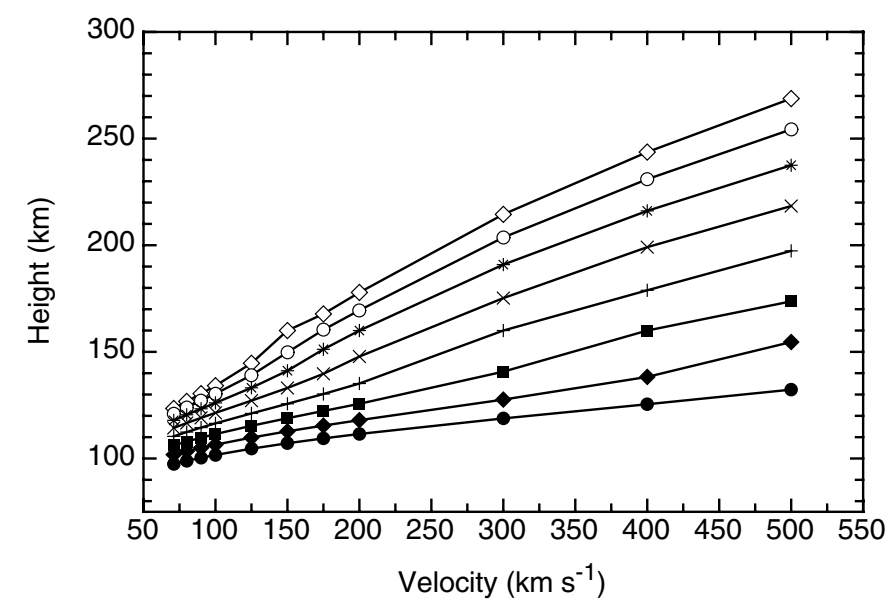

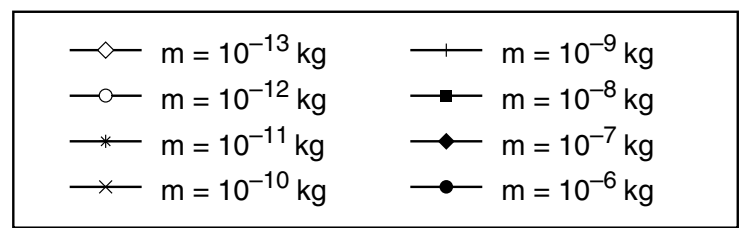

Fig. 3. Meteor heights at the point of peak light intensity $\left(H_{\max }\right)$ for asteroidal structure meteoroids.

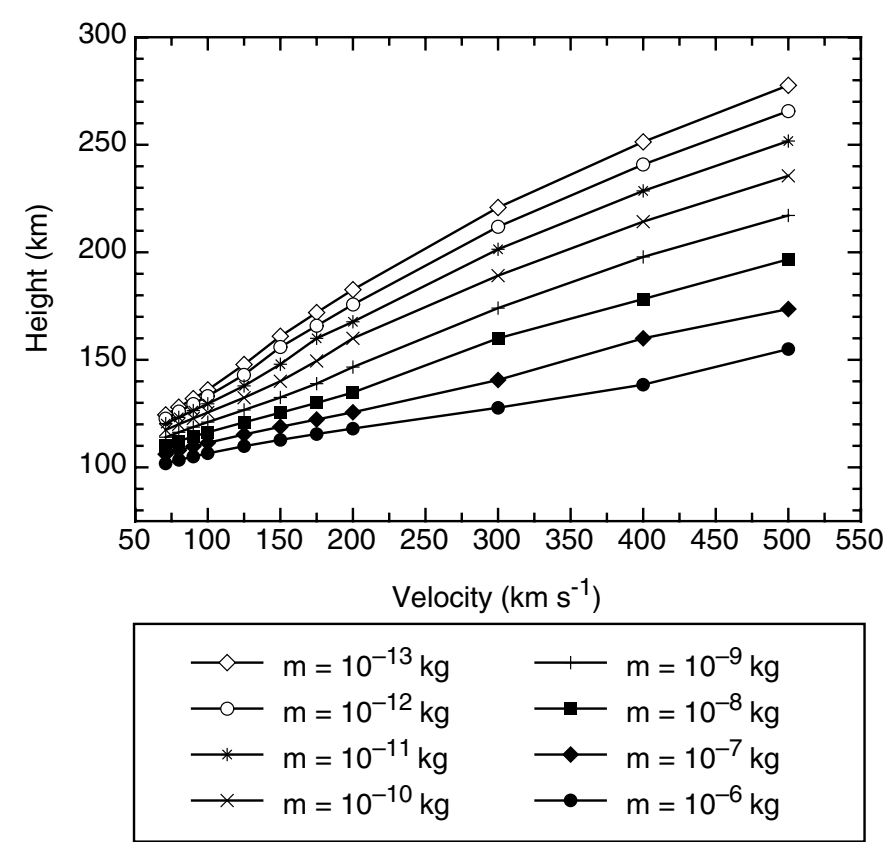

Fig. 4. Meteor heights at the point of peak light intensity $\left(H_{\max }\right)$ for cometary structure meteoroids.

of $H_{\max }$ on velocity is due to the fact that faster initial meteoroid velocities entail more energetic collisions between the meteoroid and the atmospheric constituents. Therefore, faster meteoroids do not need to travel as great a distance through the atmosphere before significant ablation begins.

The meteor heights at peak light intensity $\left(H_{\max }\right)$ also depend on the meteoroid density. By comparing Figs. 3-5, it is obvious that porous structure meteoroids consistently ablate higher than cometary structure meteoroids that in turn ablate higher than asteroidal structure meteoroids. A $10^{-10} \mathrm{~kg}$

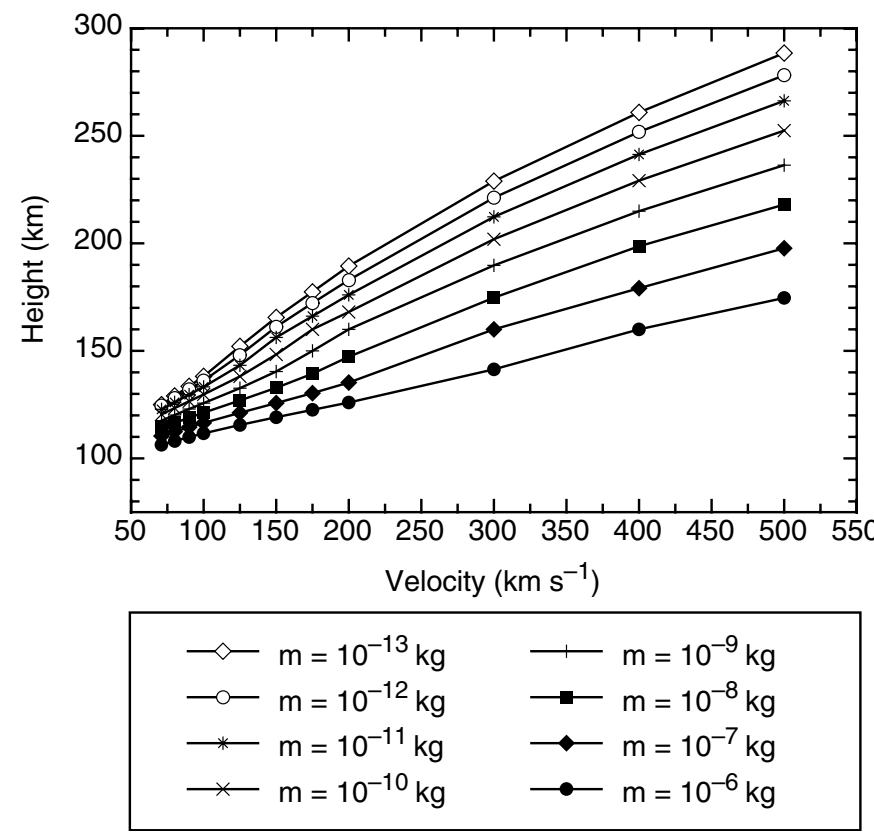

Fig. 5. Meteor heights at the point of peak light intensity $\left(H_{\max }\right)$ for porous structure meteoroids.

asteroidal meteoroid having an initial velocity of $150 \mathrm{~km} \mathrm{~s}^{-1}$ reaches its peak light intensity at a height of $133 \mathrm{~km}$ while the corresponding cometary and porous meteoroids have $H_{\max }=$ $140 \mathrm{~km}$ and $H_{\max }=148 \mathrm{~km}$ respectively. This height dependence on density is due to the fact that for a fixed mass and shape, the lower density meteoroids have a greater crosssectional area enabling more collisions with the atmospheric constituents, and therefore a larger incident kinetic energy per unit meteoroid mass.

It is often the case, especially when dealing with small, slow meteors, that meteoroid deceleration plays a significant role in reducing the rate of incident energy so that more of it can be dissipated through thermal radiation. In this study, however, deceleration was negligible in most cases and was not a dominant influence in any cases. For example, a $10^{-10} \mathrm{~kg}$ porous structure meteoroid having an initial velocity of $125 \mathrm{~km} \mathrm{~s}^{-1}$ only decelerated to $124 \mathrm{~km} \mathrm{~s}^{-1}$ at its point of peak light intensity. The meteoroid whose velocity decreased by the largest fraction was a $10^{-13} \mathrm{~kg}$ porous structure meteoroid having an initial velocity of $71 \mathrm{~km} \mathrm{~s}^{-1}$. This meteoroid had decelerated to $62 \mathrm{~km} \mathrm{~s}^{-1}$ at the point it reached its maximum light intensity. In general, less massive meteoroids encounter more significant deceleration due to a higher ratio of cross sectional area to mass.

The one magnitude vertical trail lengths $\left(\Delta H_{1}\right)$ are defined as the difference in altitude between the points before and after the meteor's maximum light intensity at which the meteor was one astronomical magnitude fainter than its peak light intensity. The two and three magnitude vertical trail lengths $\left(\Delta H_{2}\right.$ and $\left.\Delta H_{3}\right)$ are calculated in a similar manner. The one, two, and three magnitude trail lengths of asteroidal structure meteoroids are presented in Figs. 6a-6c respectively. Analogous results for cometary and porous meteoroids are presented in Figs. 7 and 8. 

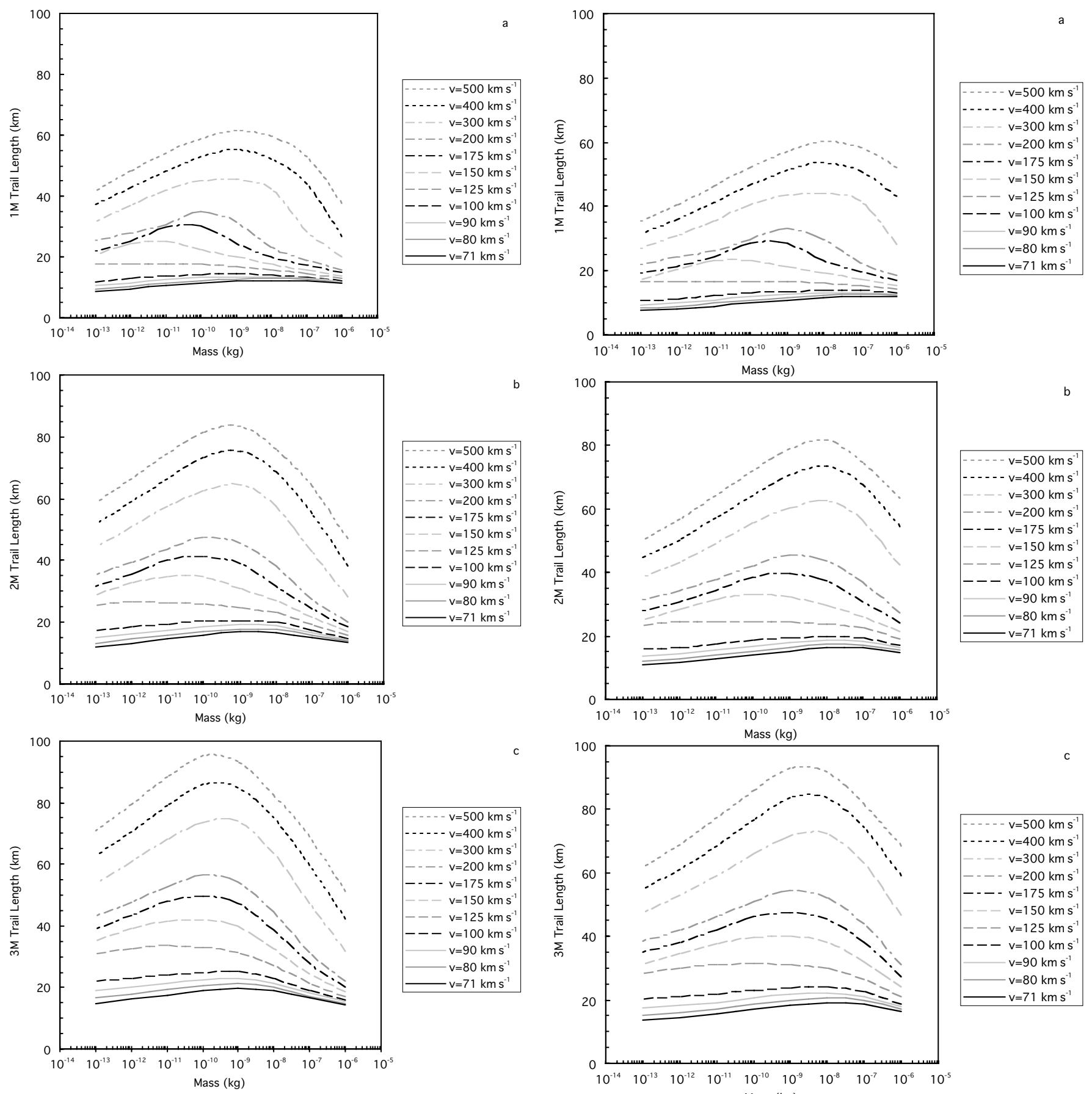

Fig. 6. a) One magnitude trail lengths $\left(\Delta H_{1}\right)$ for asteroidal structure meteoroids. Data values were calculated for masses on factors of 10 ranging from $10^{-6}$ to $10^{-13} \mathrm{~kg}$. b) Two magnitude trail lengths $\left(\Delta H_{2}\right)$ for asteroidal structure meteoroids. c) Three magnitude trail lengths $\left(\Delta H_{3}\right)$ for asteroidal structure meteoroids.

It is evident from Figs. 6-8 that with only a few exceptions (specifically in $\Delta H_{1}$ of cometary and porous meteoroids) meteoroid trail lengths increase with velocity. For instance, a $10^{-7} \mathrm{~kg}$ cometary meteoroid with an initial velocity of $100 \mathrm{~km} \mathrm{~s}^{-1}$ has $\Delta H_{2}=19 \mathrm{~km}$ while the same meteoroid having an initial velocity of $200 \mathrm{~km} \mathrm{~s}^{-1}$ has $\Delta H_{2}=37 \mathrm{~km}$. This trail length increase with velocity was to be expected since ablation begins higher for faster meteoroids where the effective

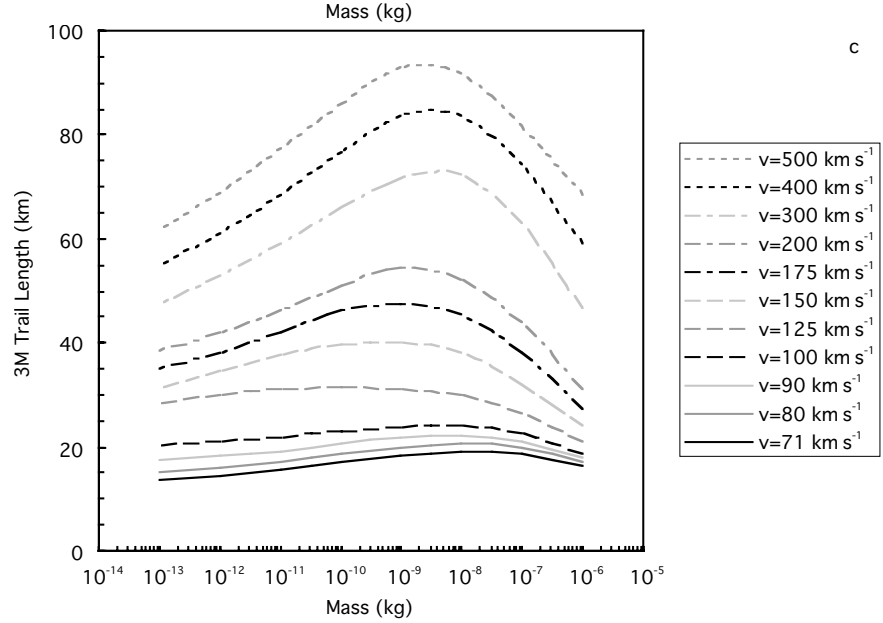

Fig. 7. a) One magnitude trail lengths $\left(\Delta H_{1}\right)$ for cometary structure meteoroids. Data values were calculated for masses on factors of 10 ranging from $10^{-6}$ to $10^{-13} \mathrm{~kg}$. b) Two magnitude trail lengths $\left(\Delta \mathrm{H}_{2}\right)$ for cometary structure meteoroids. c) Three magnitude trail lengths $\left(\Delta H_{3}\right)$ for cometary structure meteoroids.

atmospheric scale height is greater, resulting in a more spread out light curve.

Due to the mode of calculation, the trail lengths determined are highly sensitive to changes in the shapes of the meteor light curves. This may account for any anomalies in the curves plotted in Figs. 6-8. It is interesting to note that the trail lengths generally increase with mass up to a mass of approximately $10^{-10}$ to $10^{-7} \mathrm{~kg}$ before beginning to decrease. This 

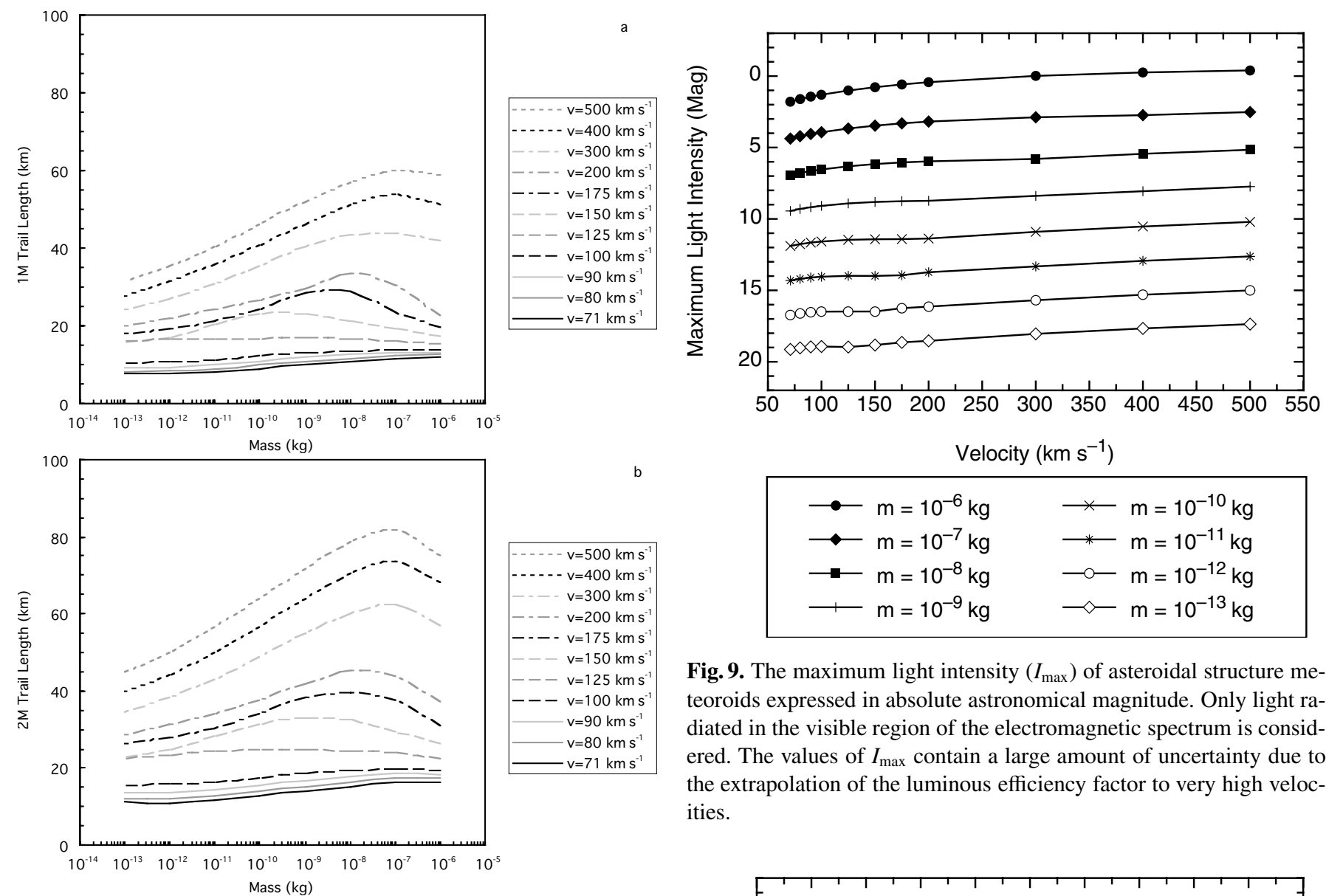

$$
\begin{array}{ll|}
\longrightarrow-\mathrm{m}=10^{-6} \mathrm{~kg} & \longrightarrow \mathrm{m}=10^{-10} \mathrm{~kg} \\
\longrightarrow \mathrm{m}=10^{-7} \mathrm{~kg} & \longrightarrow \mathrm{m}=10^{-11} \mathrm{~kg} \\
\longrightarrow \mathrm{m}=10^{-8} \mathrm{~kg} & \longrightarrow-\mathrm{m}=10^{-12} \mathrm{~kg} \\
\longrightarrow \mathrm{m}=10^{-9} \mathrm{~kg} & \longrightarrow \mathrm{m}=10^{-13} \mathrm{~kg}
\end{array}
$$

Fig. 9. The maximum light intensity $\left(I_{\max }\right)$ of asteroidal structure meteoroids expressed in absolute astronomical magnitude. Only light radiated in the visible region of the electromagnetic spectrum is considered. The values of $I_{\max }$ contain a large amount of uncertainty due to the extrapolation of the luminous efficiency factor to very high velocities.
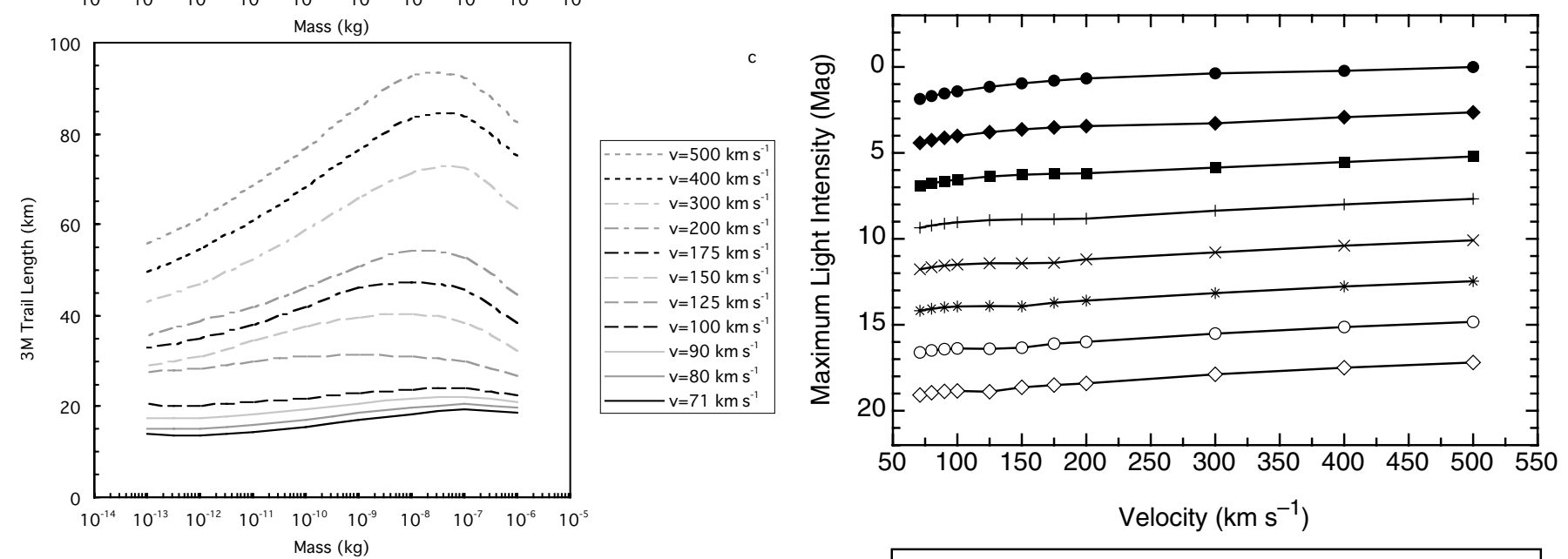

Fig. 8. a) One magnitude trail lengths $\left(\Delta H_{1}\right)$ for porous structure meteoroids. Data values were calculated for masses on factors of 10 ranging from $10^{-6}$ to $10^{-13} \mathrm{~kg}$. b) Two magnitude trail lengths $\left(\Delta H_{2}\right)$ for porous structure meteoroids. c) Three magnitude trail lengths $\left(\Delta H_{3}\right)$ for porous structure meteoroids.

behaviour is due to the competition between two processes. For small meteoroid masses, one would expect a less spread out light curve simply because less energy is required for complete ablation. At the other extreme, large meteoroids ablate lower in the atmosphere where the atmospheric density is greater, accelerating the ablation process and decreasing the trail lengths. The competition between these two effects results in maximum trail lengths observed at the mid-mass range.

Fig. 10. The maximum light intensity $\left(I_{\max }\right)$ of cometary structure meteoroids expressed in absolute astronomical magnitude.

The maximum light intensities $\left(I_{\max }\right)$ of the meteors modeled are reported in Fig. 9 (asteroidal structure), Fig. 10 (cometary structure) and Fig. 11 (porous structure). Only a very slight dependence of $I_{\max }$ on velocity is visible. Although the kinetic energy of the ablated meteoroid atoms is proportional 


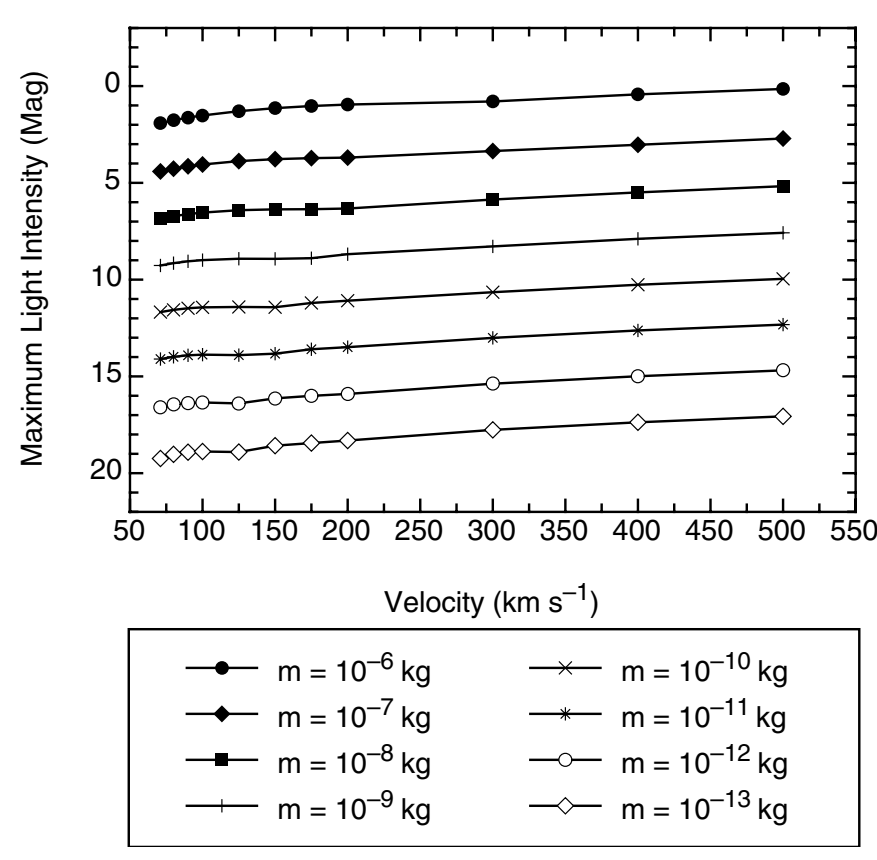

Fig. 11. The maximum light intensity $\left(I_{\max }\right)$ of porous structure meteoroids expressed in absolute astronomical magnitude.

to $v^{2}$, the extrapolated luminous efficiency decreases with velocity (see Fig. 2) yielding the observed behaviour. It is obvious from Figs. 9-11 that the meteor's peak light intensity decreases by approximately $2.5 \mathrm{mag}$ when the initial meteoroid mass increases by a factor of ten. This indicates that the light intensity (expressed in Watts) is roughly proportional to the meteoroid's initial mass. The peak light intensity $\left(I_{\max }\right)$ is observed to only vary slightly with the assumed meteoroid structure.

A great deal of uncertainty is introduced into the values of $I_{\max }$ by the extrapolation of the luminous efficiency factor $\left(\tau_{I}\right)$. It is of crucial importance in meteor physics that the variation of the luminous efficiency factor with velocity be investigated in more detail. This is especially significant to studies dealing with very fast meteoroids, as there is no experimental data available in this velocity regime. It should be stressed, however, that the trail lengths and heights of peak light intensity are largely independent of the luminous efficiency factor and are thus not so strongly subject to the same uncertainty.

Light curves for $10^{-8} \mathrm{~kg}$ asteroidal structure meteoroids with various initial velocities are displayed in Fig. 12. Similar light curves for cometary and porous structure meteoroids are reported in Figs. 13 and 14 respectively. A mass of $10^{-8} \mathrm{~kg}$ was chosen because meteoroids of this mass are sufficiently bright to be detected by sensitive electro-optical systems. It is observed that faster meteoroids radiate light over a larger range of heights. When examining the light curves presented in Figs. 12-14 it is important to bear in mind that the light intensity is expressed in absolute magnitude, and would not correspond to the apparent magnitude observed with a particular meteor range and observing system. When determining the light intensity that would be measured if a corresponding meteor were detected, the meteor height, the position of the observing station, the meteor velocity, and the attenuation of luminous

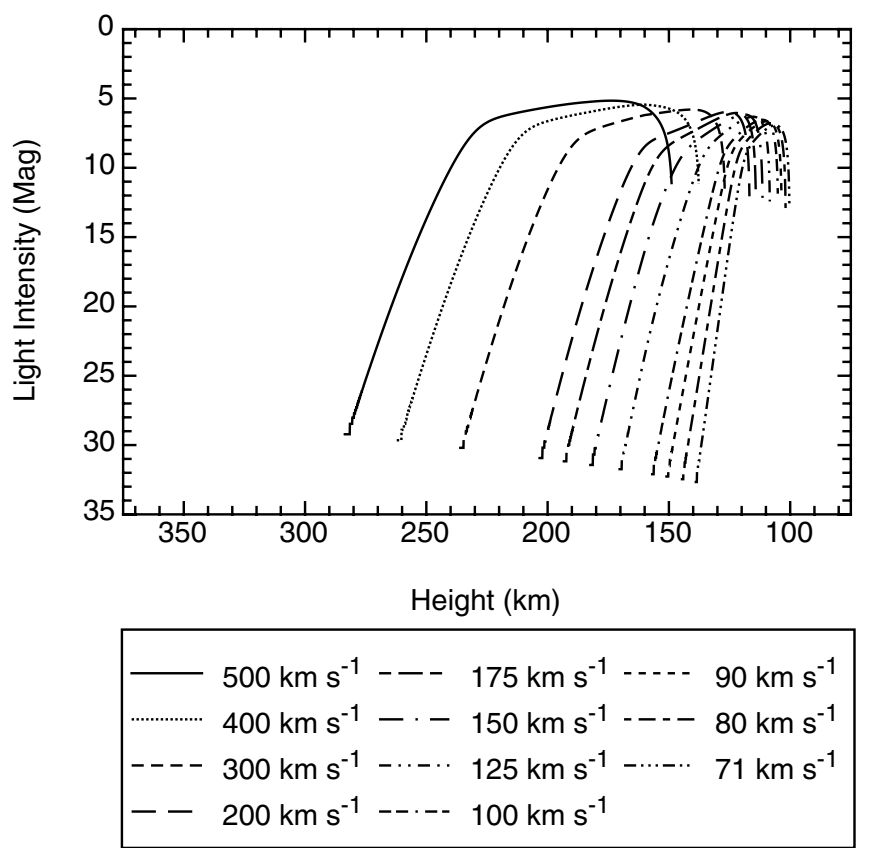

Fig. 12. Light curves for $10^{-8} \mathrm{~kg}$ asteroidal structure meteoroids with various initial velocities.

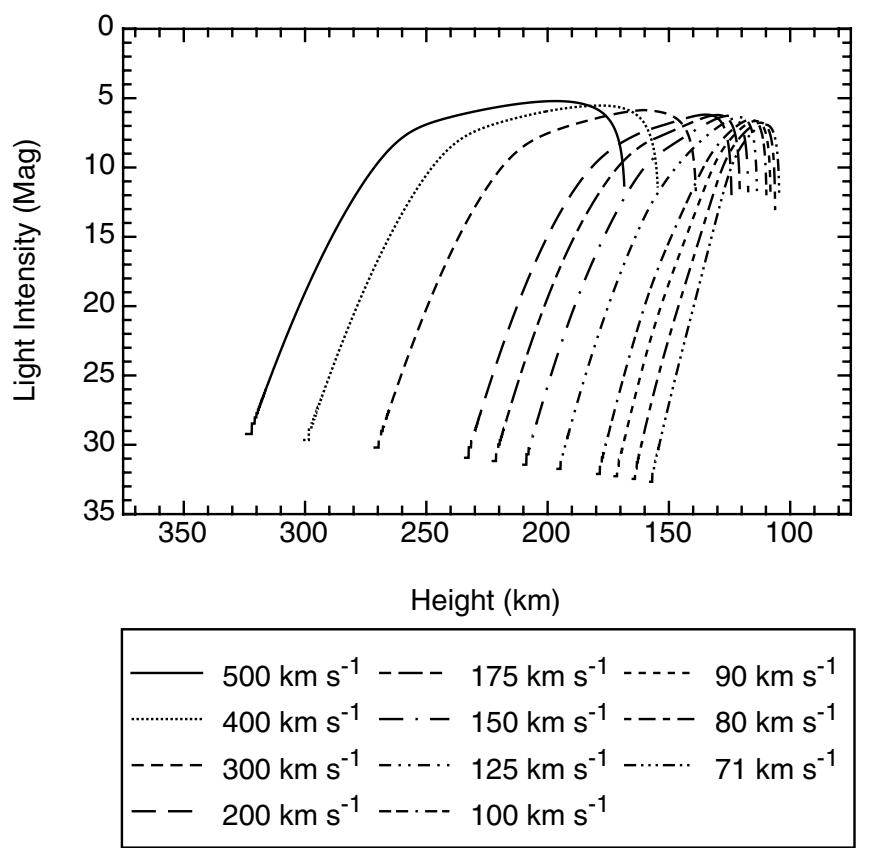

Fig. 13. Light curves for $10^{-8} \mathrm{~kg}$ cometary structure meteoroids with various initial velocities.

energy by the atmosphere must be considered. While we have provided the entire luminous light curve in these graphs, clearly the lower values are completely unobservable since they are so faint.

\section{Discussion}

In this work we have provided the heights of ablation, vertical trail lengths and expected astronomical magnitudes of high geocentric velocity meteoroids. While the brightness of these 


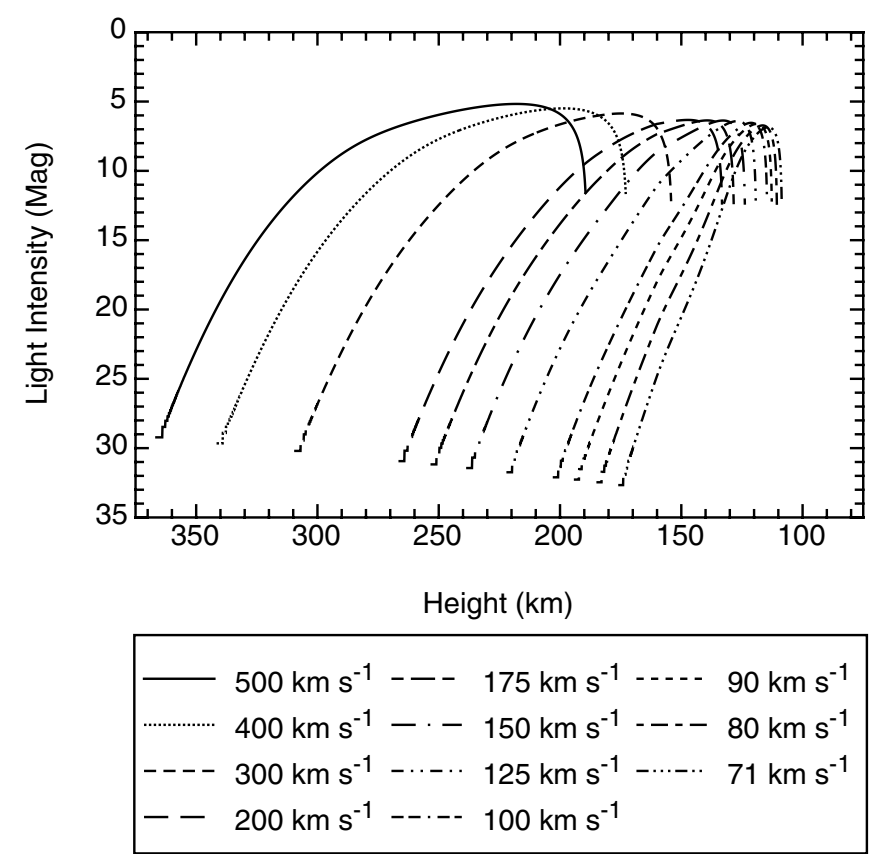

Fig. 14. Light curves for $10^{-8} \mathrm{~kg}$ porous structure meteoroids with various initial velocities.

meteors is strongly dependent on the luminous efficiency factor, and that is not well established for this velocity range, that uncertainty does not affect the trail lengths or the heights of ablation.

As noted earlier, by providing predictions of their observational characteristics our aim is to provide direction to those who seek to prove or disprove the existence of detectable numbers of high geocentric velocity interstellar origin meteoroids. The heights to be expected, in particular, could be used in optimizing a multi-station optical search for very high velocity meteors.

In general, this work suggests that very high geocentric velocity meteors could be observed with current technology if they exist. One of the somewhat surprising results is that the brightness of these meteors depends only weakly on velocity (this is primarily because the assumed luminous efficiency factor goes down with increasing velocity in this regime). However, the trail lengths become much longer with velocity. This means that the light will be spread out over a larger number of pixels on the detector (although this is partially compensated by the fact that high velocity meteors also occur at higher heights and ranges from the detector). This means that the apparent magnitudes will be reduced from the absolute magnitudes plotted in this paper due to this motion across a number of pixel elements, and there is a bias against very high geocentric velocity meteors. The correction for motion during a single frame integration time is given by Hawkes $(1998,2002)$. The larger range of these high meteors further reduces the apparent magnitude.

In Rogers et al. (2005a) we will study in detail the probability of detection of these high geocentric velocity meteors using existing image intensified CCD technology. However, except for very narrow field of view systems we would expect that these meteors could be detected in multiple frames permitting velocity determination.

As mentioned in the introduction, there is no well established optical detection of a very high geocentric velocity meteor, although the observational sample is small. It should be pointed out that in multi-station observing campaigns with triangulation used to establish meteor trajectories and orbits there will be an observational bias according to the optimum intersection height assumed. Woodworth \& Hawkes (1996) have performed a series of simulations in order to predict the significance of this bias. Their results show that the bias is very significant for narrow field of view systems, particularly when they look in directions well offset from the vertical. In another consideration of this bias, Campbell et al. (2000) considered the effect of the range on apparent magnitude, as well as the loss of probability of simultaneous detection. They found that for very large fields of view the effect is not strong. The results reported in this paper suggest that the apparent magnitudes of these meteors may be moderately faint. Unfortunately, detection of these meteors would require large apertures, normally related to longer focal lengths, and thus smaller fields of view with stronger biases. It should be kept in mind that the effective collecting area of narrow field of view systems is significantly more than the geometric collecting area (Kresakova 1977).

The observational case for radar detections of very high velocity meteors is not clear. It appears that there are a few very high velocity meteors observed with systems such as AMOR, but the measurement errors also are very large for these meteors. In terms of predictions, while the ionizing efficiency goes up strongly with meteor velocity for moderate geocentric velocities, the dependence of ionizing efficiency at very high geocentric velocities is not clear. Jones (1997) has developed theoretical relationships for predicting the ionization coefficient for moderate velocities, which may possibly be extrapolated into the regime studied in this paper. The rapid diffusion of the ionization column at great heights severely diminish the radar return unless powerful radars with relatively large wavelengths are used (McKinley 1961). Fragmentation may further weaken the radar return (Campbell \& Jones 2001), and the physical nature of interstellar origin meteoroids is not known. Most solar system meteoroids in this size range probably have a dustball structure (e.g. Fisher et al. 2000).

Acknowledgements. This work was funded by Discovery Grants to RLH from the Natural Sciences and Engineering Research Council (NSERC) of Canada. KAH and LAR were supported by NSERC Undergraduate Research Awards as well. We would like to acknowledge the very helpful comments of the two referees, Pavel Koten and Detlef Koschny.

\section{References}

Adolfsson, L. G., Gustafson, B. À. S., \& Murray, C. D. 1996, Icarus, 119,144

Baggaley, W. J. 2000, J. Geophys. Res., 105, 10353

Baggaley, W. J., \& Neslusan, L. 2002, A\&A, 382, 1118

Becker, D. G., \& Friichtenicht, J. F. 1971, ApJ, 166, 699

Boinott, C. A., \& Savage, H. F. 1972, ApJ, 174, 201

Bronshten, V. A. 1983, Physics of Meteor Phenomena (Dordrecht: D. Reidel) 
Brophy, T. G. 1991, Icarus, 94, 250

Campbell, M. D., Brown, P. G., LeBlanc, A. G., et al. 2000, MAPS, 35,1259

Campbell, M. D., \& Jones, J. 2001, in Meteoroids, ed. B. Warmbein, ESA SP-495, 265

Ceplecha, Z., Borovicka, J., Elford, W. G., et al. 1998, Space Sci. Rev., 84,327

Ellyett, C. 1977, J. Geophys. Res., 82, 1455

Fisher, A. A., Hawkes, R. L., Murray, I. S., Campbell, M. D., \& Leblanc, A. G. 2000, Planet. Space Sci., 48, 911

Friichtenicht, J. F., \& Becker, D. G. 1973, in Evolutionary and Physical Properties of Meteoroids, ed. C. L. Hemingway, 53

Hawkes, R. L. 1998, Highlights in Astron., 11, 1017

Hawkes, R. L. 2002, in Meteors in the Earth's Atmosphere, ed. I. P. Williams, \& E. Murad (Cambridge University Press), 97

Hawkes, R. L., Close, T., \& Woodworth, S. C. 1999, in Meteoroids, ed. I. P. Williams, \& V. Porubcan, 257

Hawkes, R. L., \& Jones, J. 1975, MNRAS, 173, 339

Hawkes, R. L., \& Woodworth, S. C. 1997, J. Roy. Astr. Soc. Canada, 91, 68

Hedin, A. E. 1987, J. Geophys. Res., 92, 4649

Hedin, A. E. 1991, J. Geophys. Res., 96, 1159

Jones, W. 1997, MNRAS, 288, 995

Jones, W., \& Halliday, I. 2001, MNRAS, 320, 417
Jones, A. P., Tielens, A. G. G. M., Hollenbach, D. J., \& McKee, C. F. 1997, in Astrophysical Implications of the Laboratory Study of Presolar Materials, ed. T. J. Bernatowicz, \& E. Zinner, 595

Kresakova, M. 1977, Bull. Astron. Inst. Czech., 28, 340

Krivova, N. A., \& Solanki, S. K. 2003, A\&A, 402, L5

McKinley, D. W. R. 1961, Meteor Science and Engineering (Toronto: McGraw Hill)

Murray, N., Weingartner, J., \& Capobianco, P. 2004, ApJ, 600, 804

Nicol, E. J., Macfarlane, J., \& Hawkes, R. L. 1985, Planet. Space Sci., 33, 315

Öpik, E. J. 1958, Physics of Meteor Flight in the Atmosphere (New York: Interscience)

Quirt, B., \& Hawkes, R. L. 2005, Planet. Space Sci., submitted

Rogers, L. A., Hill, K. A., \& Hawkes, R. L. 2005a, Earth Moon Planets, 95, in press

Rogers, L. A., Hill, K. A., \& Hawkes, R. L. 2005b, Planet. Space Sci., 53, 1341

Šimek, M., \& Pecina, P. 2002, Earth Moon Planets, 88, 115

Taylor, A. D., Baggaley, W. J., \& Steel, D. I. 1996, Nature, 380, 323

Verniani, F. 1965, Smithson. Contrib. Astrophys., 8, 141

Whipple, F. L. 1938, Proc. Am. Phil. Soc., 79, 499

Woodworth, S. C., \& Hawkes, R. L. 1996, in Physics, Chemistry and Dynamics of Interplanetary Dust, ed. B. Ȧ. S. Gustafson, \& M. S. Hanner, ASP, 104, 83 